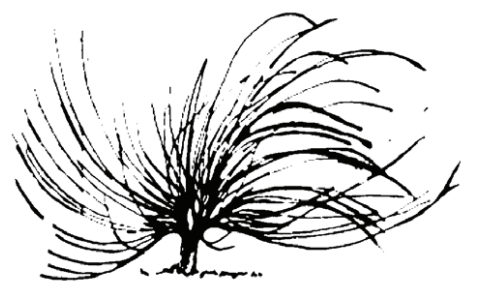

\title{
Evaluar Competencias a Partir de la Autenticidad: Implicaciones en la Docencia Universitaria
}

\author{
Giovanni Sánchez Chacón ${ }^{1}$ \\ Universidad Nacional \\ Heredia, Costa Rica \\ giovanni.sanchez.ch@gmail.com
}

\begin{abstract}
Resumen
En el presente ensayo, se argumenta la relevancia del paradigma de la evaluación auténtica como plataforma ideal para la evaluación de competencias profesionales en la docencia universitaria. Para estos efectos, se reflexiona brevemente en torno al concepto de competencias y sus eventuales aportes a la educación superior, para posteriormente argumentar la funcionalidad del paradigma de la autenticidad, de cara a la evaluación de competencias profesionales.
\end{abstract}

Palabras clave: evaluación, medición, evaluación auténtica, competencias profesionales, docencia universitaria

(c) $(7)(\$$

Recibido: 10 de febrero de 2014-Aprobado: 13 de abril de 2015

1 Docente en Centro de Investigación y Docencia en Educación, CIDE, UNA. Director de Docencia, Universidad Santa Paula, Costa Rica. Bachiller en Música con concentración en Educación Musical: Universidad Nacional, Costa Rica. Licenciado en Ciencias de la Educación con énfasis en Didáctica de la Música: Universidad Nacional, Costa Rica. Máster en Psicopedagogía, Universidad Estatal a Distancia: UNED, Costa Rica. Máster en Musicoterapia: Instituto Superior de Estudios Psicológicos de Barcelona, España. Posgrado en Psicología Evolutiva y de la Educación: Universidad Autónoma de Barcelona, España. Doctor en Psicología de la Educación: Universidad Autónoma de Barcelona, España. 


\begin{abstract}
In this essay, the author argues about the relevance of the authentic assessment paradigm as an ideal platform for the assessment of professional competence in university teaching. To this end, the author briefly discusses the concept of competence and its possible contributions to higher education to further argue about the functionality of the authenticity paradigm in the professional competence assessment.
\end{abstract}

Keywords: assessment, measurement, authentic assessment, professional competencies, university teaching

\title{
Introducción
}

$\mathrm{E}$ 1 presente ensayo pretende desarrollar una reflexión en torno a los aportes de la evaluación auténtica, como iniciativa metodológica para evaluar competencias profesionales, concretamente en la docencia universitaria. Desde esta óptica, la investigación especializada ha señalado en los últimos años, la necesidad de introducir diversas formas de evaluación en educación superior, que permitan la formación de un ser humano integral, a través de la sólida construcción de competencias personales y profesionales y no de simples saberes aislados de sus diversos contextos reales de aplicación. Para estos efectos, se argumentará, por qué los principios de la evaluación auténtica, podrían aportar un marco conceptual y metodológico de interés, que conduzca a una evaluación auténtica y situada en las competencias profesionales, en donde esta sea concebida como un proceso más de aprendizaje para el estudiante y de toma de decisiones para el docente universitario.

\section{Reflexión en torno al concepto de competencias}

Vinculado estrechamente al ámbito de la formación profesional, el concepto de competencias ha venido a asumir un relevante protagonismo en los últimos 20 años, distinguiéndose en este ámbito, una gran cantidad de propuestas curriculares y posicionamientos teóricos. De acuerdo con (Coll, 2007) el discurso de las competencias, ha ido ganando terreno de forma progresiva en todos los ámbitos y niveles de la educación formal, desde la educación superior hasta la educación infantil, convirtiéndose en muchos países en un enfoque dominante. 
Sin afán de limitar las implicaciones metodológicas de este concepto, al desarrollo de prácticas educativas meramente instrumentales, es preciso señalar que su implementación desde una perspectiva sociocultural y situada en la docencia universitaria, nos permite realizar una reflexión en torno a cuáles son los saberes disciplinares que un sujeto debe aprender, su forma de organización jerárquica y su respectiva articulación existente entre ellos en diversos escenarios de aplicación. En este sentido, Coll (2007) señala que el interés fundamental del concepto de competencia reside en el hecho de que proporciona una mirada muy sugerente para abordar un aspecto extremadamente complejo de la educación: la identificación, selección, caracterización y organización de los aprendizajes; es decir, las decisiones relativas a lo que debe esforzarse en aprender el alumnado y, por tanto, a lo que debe intentar enseñar el profesorado.

Desde una perspectiva general, Zabala y Arnau (2007) y Monereo (2007) sostienen que el empleo de una competencia, supone la articulación y movilización de manera coordinada, de un repertorio de estrategias o saberes que se ponen en marcha para responder eficazmente a una demanda o problema prototípico, del contexto social, interpersonal, personal y profesional. Tomando en consideración estas palabras y llevándolas al contexto de la enseñanza y aprendizaje en la universidad, podríamos afirmar que desde una perspectiva sociocultural y situada de la docencia universitaria, la enseñanza de una competencia supone el empleo de múltiples estrategias por parte del docente, con el propósito de desarrollar movilidad y coordinación en la ejecución de un repertorio de saberes que el futuro profesional debe poner en marcha para responder eficazmente a unas demandas concretas del contexto profesional.

Por lo anterior, el concepto de competencias debe ser abordado desde una perspectiva auténtica y situada, ya que ellas se deben enseñar y evaluar a partir de sus condiciones y escenarios reales de aplicación.

Debido al papel protagónico que los modelos por competencias han tomado y a la necesidad de diseñar iniciativas pedagógicas que permitan su adecuada evaluación, en los últimos años, se ha destacado un creciente interés por concebir a la evaluación auténtica como un paradigma que reúne el componente epistemológico y metodológico adecuado para dicha tarea. Estos planteamientos han sido propuestos por Dominique et al. (2008) y a nivel iberoamericano (Monereo, 2007). 
En este sentido, algunas de las relaciones entre ambos paradigmas, se visualizan claramente en las aportaciones realizadas por Zabala y Arnau (2007), quienes proponen que enseñar competencias, supone partir de situaciones y problemas del mundo real, los cuales tienen lugar en el contexto social, interpersonal, personal y profesional del sujeto. Por su parte, Coll (2009) propone una serie de principios, que a su parecer, justifican y ponen en evidencia las ventajas de una formación basada en competencias, en contraposición a otras teorías y modelos de aprendizaje. Dichos principios están muy relacionados con las bases conceptuales y metodológicas de la evaluación auténtica que veremos en apartados siguientes. A continuación, se hace referencia a las ventajas de una formación basada en competencias señalada por Coll (2009).

a. Funcionalidad. La enseñanza por competencias permite que todo contenido por enseñar posea una clara funcionalidad en su contexto habitual de aplicación. De tal manera, que el docente no solamente se limitará a modelar los conceptos, sino que intentará paralelamente enseñar cuál es la funcionalidad de ellos en la práctica profesional real.

b. Articulación. La enseñanza por competencias permite que al enseñar un nuevo contenido, también se enseñe como este se articula con otros ya estudiados y como de manera coordinada se movilizan otros saberes para dar respuesta a una demanda del contexto profesional.

c. Contexto de aplicación. En este paradigma, se asume que al enseñar un determinado contenido o tema, es necesario que el estudiante tenga la posibilidad de practicar el aprendizaje logrado en contextos reales en los que habitualmente dichos saberes deban ser puestos en marcha.

Desde esta óptica, una enseñanza basada en competencias aporta relevancia y atribución de sentido y significado del aprendizaje, ya que sugiere una educación para la vida, en donde es posible de manera inmediata, encontrar un sentido de utilidad a lo que se aprende. Para estos efectos, podemos afirmar que los principios de la autenticidad, representan una opción metodológica viable para la evaluación de competencias profesionales, tal y como lo establecen las bases conceptuales de la evaluación auténtica, en las cuales profundizaremos a continuación. 


\section{Bases conceptuales y metodológicas de la Evaluación Auténtica}

De acuerdo con Fernández (2009), desde hace aproximadamente dos décadas, la investigación especializada viene señalando una serie de planteamientos evaluativos centrados en una evaluación de los aprendizajes orientada hacia la evaluación del proceso y altamente preocupada por el aprendizaje a través de tareas auténticas, en donde los eventuales formatos y situaciones / problemas por evaluar, están relacionados con el contexto profesional, en el caso particular de la educación superior. Por ello, los resultados poco alentadores obtenidos a través de las pruebas estandarizadas tradicionales, se han convertido en la base para la toma de decisiones en materia de evaluación de los aprendizajes. De acuerdo con Woolfolk (1999), esta situación se debe a la política educativa tradicional, que apuesta a una valoración de los aprendizajes, a partir de los resultados cuantitativos obtenidos a través de un examen escrito. Esta concepción posee mayor afinidad al uso de técnicas instrumentales que apuestan a la simple medición de los aprendizajes y no hacia el avance que representa migrar hacia nuevos paradigmas que permitan valorar con mayores argumentos como ha sido el proceso global de construcción del conocimiento de un sujeto.

Como se ha sostenido, evaluar competencias permite apreciar el valor completo de un fenómeno o de un producto, por lo tanto, se podría considerar que existe un proceso de evaluación de competencias, cuando se justifica desde una perspectiva amplia y argumentada, por qué un estudiante requiere de ayudas pedagógicas concretas o es competente en un determinado tema, en general, implica acceder a detalles del cómo el alumno ha construido su conocimiento, lo cual sin duda, permite una mejor toma de decisiones al profesorado. Al respecto, Fernández (2009) sostiene que una evaluación de competencias, se basa en el acceso a múltiples fuentes de información con el propósito de conocer si los estudiantes han alcanzado el dominio de los diversos recursos y saberes vinculados a cada competencia.

Desde esta perspectiva, es innegable que nuestro sistema educativo, en todos sus niveles, apuesta por una evaluación centrada en la medición, la cual se sigue reproduciendo con cierta frecuencia en la educación superior. Dicha situación, pone en evidencia el escaso impacto que las tendencias contemporáneas socioconstructivistas y de carácter situado, han generado en las concepciones epistemológicas del profesorado en materia de evaluación de los aprendizajes. 
Paralelamente a estos precedentes, en la actualidad, existe una creciente tendencia a concebir la evaluación, como aquella estructura que configura el entramado pedagógico que gira en torno a un proceso educativo. Si esta premisa es correcta, se infiere que las innovaciones educativas dirigidas a la transformación progresiva de las estrategias de evaluación, muy probablemente podrían ser el motor que impulse cambios en las prácticas educativas de los docentes, incluso en los diversos modos de enfocar y autorregular el estudio por parte de los estudiantes (Gibbs, 1992; Wolf et al., 1991). Al respecto, Prodromou (1995) y más recientemente Monereo (2009), han señalado la influencia decisiva de la evaluación, en las prácticas educativas de los docentes, incluso sobre las decisiones relacionadas en cuanto a cuál o cuáles contenidos deben ser evaluados con mayor rigurosidad en un proceso educativo.

Las reflexiones anteriores, nos llevan a inferir que la evaluación podría representar uno de los ejes primordiales para el cambio educativo, con mucha más razón, de cara a la docencia universitaria, donde debería concebirse como una plataforma ideal para el desarrollo de verdaderas competencias profesionales, en las que la memoria y las interpretaciones numéricas representarían tan solo un aspecto de una gran cantidad de saberes y formas constructivistas de evaluación necesarias de cara a la formación profesional. Debido a lo anterior, en la actualidad, se debate si la introducción de cambios en la forma de concebir y llevar a la práctica la evaluación en la educación superior, podría conllevar repercusiones favorables en la mejora de las prácticas educativas y de manera muy particular, en la calidad de lo que realmente aprende el estudiante universitario.

Por esta razón, emerge la expresión evaluación auténtica, la cual suele emplearse para describir una diversidad de nuevos enfoques, principios e instrumentos de evaluación con una orientación muy distinta a los utilizados comúnmente en la evaluación tradicional (Monereo, 2009). Al respecto Savery y Duffy (1995), definen la autenticidad de una tarea o actividad, como la similitud o coherencia existente entre las condiciones que dicha evaluación plantea y las existentes en el contexto real en el que deben ser puestos en marcha los contenidos evaluados.

En esta misma línea, Gielen, Dochy y Dierick (2003) sugieren que al proponer una evaluación auténtica, han de tomarse en consideración dos criterios relevantes, los cuales consisten en la validez de constructo y la validez consecuencial. La validez de constructo, se centraría 
en la necesidad de valorar la naturaleza misma de lo que se pretende medir, es decir, se debe enseñar y evaluar a partir de problemas reales que guarden una cierta dosis de autenticidad y relación con eventuales situaciones profesionales en las que un estudiante deberá hacer uso de lo aprendido. Por otra parte, la validez consecuencial está relacionada con el nivel de coherencia existente entre la forma y nivel de dificultad con que se explica y enseña un determinado tema y el nivel de exigencia demandado en la evaluación. Los anteriores principios nos conducen a interpretar que una evaluación es auténtica, en la medida que exista una fidelidad palpable entre la actividad de evaluación presentada a los estudiantes y los diversos escenarios y contextos profesionales en que los contenidos evaluados deban ponerse en marcha.

Lo expuesto corrobora la gran diferencia que existe entre los principios sobre los cuales se articula la evaluación tradicional y la evaluación auténtica. Al respecto, (Wiggins, 1990) señala las principales diferencias entre una y otra, las cuales se exponen a continuación en la tabla 1:

Tabla 1. Diferencias entre evaluación tradicional y auténtica (Wiggins, 1990).

\begin{tabular}{|l|l|}
\hline \multicolumn{1}{|c|}{ Evaluación tradicional } & \multicolumn{1}{c|}{ Evaluación Auténtica } \\
\hline $\begin{array}{l}\text { La evaluación gira en torno a ítems } \\
\text { indirectos. }\end{array}$ & $\begin{array}{l}\text { Se enfatiza en la ejecución del estudiante, } \\
\text { con base en tareas relevantes y } \\
\text { funcionales. }\end{array}$ \\
\hline $\begin{array}{l}\text { Enfatiza en aprendizajes adquiridos } \\
\text { solamente en las aulas. }\end{array}$ & $\begin{array}{l}\text { Los estudiantes aplican lo aprendido en } \\
\text { situaciones de la vida cotidiana. }\end{array}$ \\
\hline $\begin{array}{l}\text { Las evaluaciones tradicionales suelen ser } \\
\text { de "lápiz y papel" y las preguntas por lo } \\
\text { general suponen una única respuesta. }\end{array}$ & $\begin{array}{l}\text { Evalúa simultáneamente un conjunto de } \\
\text { competencias necesarias para resolver } \\
\text { situaciones relevantes del contexto } \\
\text { profesional. }\end{array}$ \\
\hline $\begin{array}{l}\text { Suelen considerarse solo los resultados. } \\
\text { Se requiere la justificación argumentada } \\
\text { de respuestas y comportamientos. }\end{array}$ \\
\hline $\begin{array}{l}\text { Con frecuencia la validez se asume a } \\
\text { través del apareamiento de ítems con el } \\
\text { contenido del currículum }\end{array}$ & $\begin{array}{l}\text { Se evalúa la capacidad de actuar en } \\
\text { múltiples contextos sociales eficazmente. }\end{array}$ \\
\hline $\begin{array}{l}\text { Se evalúan elementos estáticos, y } \\
\text { arbitrarios, que pueden resolverse } \\
\text { casi siempre a través de la aplicación } \\
\text { mecánica de principios y formulaciones. }\end{array}$ & $\begin{array}{l}\text { Se emplean problemas mal estructurados } \\
\text { y en ocasiones ambiguos, que reflejan la }\end{array}$ \\
\hline
\end{tabular}


Como se aprecia en la tabla 1, las diferencias entre una y otra postura son notorias, como serían también las implicaciones que cada postura posee en el aprendizaje del estudiante. Por ello, lo anterior nos conduce a reflexionar en torno al hecho de que es lo que hace auténtica a una tarea o actividad de evaluación. Monereo (2009) nos sugiere algunos insumos al respecto.

\section{Análisis de la Autenticidad de una tarea de evaluación}

Los expuesto en párrafos anteriores, nos lleva a concluir que en ocasiones, no todo lo que se evalúa es relevante o funcional para los estudiantes, es decir, no todos los procesos de evaluación, cumplen con las exigencias conceptuales y metodológicas para que dicha evaluación sea auténtica. Monereo (2009) propone una serie de criterios a partir de los cuales se podría identificar el nivel de autenticidad de una actividad de evaluación. Desde la perspectiva de dicho autor, una actividad de evaluación sería más o menos auténtica cuando:

a. Nivel de realismo es elevado. Cuando las condiciones de aplicación de la actividad evaluativa y sus exigencias cognitivo / procedimentales, son muy similares a las condiciones y exigencias implicadas en la ejecución de dicha actividad en su contexto profesional habitual.

b. Nivel de relevancia elevado. Los aprendizajes que conlleva la actividad de evaluación, deben ser directamente útiles para que el alumno enfrente situaciones y problemas reales de su vida personal y profesional.

c. Proximidad eclógica elevada. Se refiere a que la autenticidad, debe irse propiciando a partir de iniciativas que forman parte de la cotidianidad de los docentes y de la cultura institucional, es decir, es importante que dichas innovaciones en materia de evaluación, no sean introducidas de manera muy artificial desde afuera, lo cual haga que los docentes y la misma institución, no se identifiquen con dichas innovaciones.

d. Alta implicación identitaria. Apunta a que las actividades planteadas, deben evaluar al alumno en situaciones que supongan distintos grados de inmersión profesional, según el nivel de avance en el plan de estudios cursado y la familiarización con ciertos 
problemas prototípicos y emergentes propios del contexto profesional, que en una determinada fase de su formación, los estudiantes estarían en posición de enfrentar.

Una vez teniendo claro el concepto de autenticidad y de cuáles podrían ser los criterios para valorar si una tarea o actividad de evaluación es auténtica, pasaremos a hacer referencia a una serie de metodologías señaladas por la investigación especializada y su respectiva dosificación a lo largo de un eventual proceso de formación profesional. En relación con algunas de sus bases metodológicas, se podrían diseñar estrategias y escenarios que permitan evaluar competencias a través de los principios de la autenticidad.

\section{Métodos de Evaluación Auténtica: transición entre la comunidad escolar y profesional.}

Uno de los factores por considerar al intentar autentificar un proceso de evaluación, consiste en ser conscientes del nivel de conocimientos y el grado de inmersión profesional con el que los estudiantes asumen la actividad de evaluación planteada. Un intento de autentificación poco planificado e ingenuo, podría generar incertidumbre entre los estudiantes, al sentirse superados por las demandas de la situación evaluativa planteada, lo cual, lejos de generar beneficios académicos, podría tener un impacto negativo sobre los aprendizaje de ellos (Sweller, Van Merriënboer y Paas, 1998).

Desde esta óptica, Huang y Der-Thanq (2007), citados por Monereo (2009), proponen un conjunto de métodos de evaluación que eventualmente podrían favorecer esa transición existente entre la comunidad educativa (primeros años de formación profesional) y la comunicad profesional (últimos años de formación profesional e inserción al mercado laboral). Dichos métodos son coherentes con los principios de la evaluación auténtica y no pretenden ser concebidos como iniciativas rígidas, mecánicas y meramente instrumentales, sino como pautas generales y ejemplos acerca de cómo podríamos recrear diversas formas y contextos de evaluación cada vez más auténticos a lo largo de un proceso de formación profesional. Cabe señalar que dichas metodologías representan tan solo la situación o actividad de aprendizaje sobre la cual se articulará un proceso de evaluación y que las mismas deberán 
ser acompañadas por rúbricas cualitativas que permitan al docente identificar y argumentar como se articulan los saberes implicados en cada competencia a evaluar. Es preciso que dichas rúbricas, sean diseñadas en función de la especialidad y de las competencias que se pretenden evaluar. A continuación, en la tabla 2 se realiza una breve referencia de dichas metodologías:

\section{Tabla 2. Autenticidad y Transición entre la comunidad escolar y profesional (Huang y Der-Thanq, 2007).}

\begin{tabular}{|c|c|c|}
\hline Metodología & Definición & Ejemplo \\
\hline a) Extrapolación & $\begin{array}{l}\text { Consiste en un conjunto de } \\
\text { metodologías de evaluación } \\
\text { en las que se propone la } \\
\text { enseñanza y aplicación de } \\
\text { conceptos propios de una } \\
\text { disciplina, a problemas y } \\
\text { demandas del "mundo real". }\end{array}$ & $\begin{array}{l}\text { Evaluación de conceptos } \\
\text { disciplinares a través de su } \\
\text { aplicación en la resolución de } \\
\text { casos prácticos. }\end{array}$ \\
\hline b) Emulación & $\begin{array}{l}\text { Consiste en la reproducción } \\
\text { de escenarios y prácticas } \\
\text { del contexto profesional en } \\
\text { los cuales el alumno puede } \\
\text { experimentar o ser evaluado. }\end{array}$ & $\begin{array}{l}\text { Prácticas de evaluación } \\
\text { diseñadas a partir de la } \\
\text { simulación de problemas } \\
\text { derivados del contexto } \\
\text { profesional. }\end{array}$ \\
\hline c) Co-apropiación & $\begin{array}{l}\text { Estos parten de actividades } \\
\text { de enseñanza y evaluación } \\
\text { en las que debe resolverse un } \\
\text { problema auténtico propio } \\
\text { de la disciplina, a través de } \\
\text { la cooperación en parejas } \\
\text { o en grupos pequeños de } \\
\text { estudiantes. }\end{array}$ & $\begin{array}{l}\text { Resolución de casos reales en } \\
\text { equipos de trabajo o parejas, en } \\
\text { donde cada miembro posee un } \\
\text { rol claro. }\end{array}$ \\
\hline d) Extensión & $\begin{array}{l}\text { Se suelen introducir en clase } \\
\text { elementos propios de la cultura } \\
\text { profesional, con el propósito } \\
\text { de propiciar un acercamiento } \\
\text { del estudiante a dicha cultura. }\end{array}$ & $\begin{array}{l}\text { Uso de herramientas propias } \\
\text { de la disciplina en el análisis } \\
\text { de casos reales, resolución de } \\
\text { incidentes críticos vinculados a } \\
\text { la profesión, etc. }\end{array}$ \\
\hline e) Restricción & $\begin{array}{l}\text { Este grupo representa tareas } \\
\text { o actividades profesionales } \\
\text { reales, pero de menor } \\
\text { complejidad y con supervisión } \\
\text { profesional. }\end{array}$ & $\begin{array}{l}\text { Actividades que supongan la } \\
\text { colaboración de un profesional } \\
\text { del ámbito, en tareas } \\
\text { profesionales sencillas. }\end{array}$ \\
\hline
\end{tabular}




\begin{tabular}{|l|l|l|}
\hline \multicolumn{1}{|c|}{ Metodología } & \multicolumn{1}{|c|}{ Definición } & \multicolumn{1}{c|}{ Ejemplo } \\
\hline f) Participación: & $\begin{array}{l}\text { Se proponen estrategias } \\
\text { evaluativas que supongan } \\
\text { situar al estudiante en el } \\
\text { contexto profesional de manera } \\
\text { activa y comprometida, } \\
\text { ejerciendo tareas plenamente } \\
\text { profesionales, igualmente bajo } \\
\text { la supervisión de un tutor. }\end{array}$ & $\begin{array}{l}\text { Dichas actividades podrían } \\
\text { establecerse en las etapas } \\
\text { finales de un proceso de } \\
\text { formación profesional, } \\
\text { tomando como plataforma la } \\
\text { práctica profesional. }\end{array}$ \\
\hline g) Co-evolución & $\begin{array}{l}\text { Este tipo de tareas, implican } \\
\text { la realización de un trabajo } \\
\text { plenamente profesional, en } \\
\text { colaboración con otros colegas, } \\
\text { compartiendo los distintos } \\
\text { procesos de intervención y } \\
\text { desarrollando un papel activo } \\
\text { en el que se asuma un rol claro } \\
\text { que aporte al resto del equipo } \\
\text { de trabajo. }\end{array}$ & $\begin{array}{l}\text { También esta se puede } \\
\text { abordar en espacios como } \\
\text { la práctica profesional, al } \\
\text { plantear al estudiante su } \\
\text { participación en algún equipo } \\
\text { o comisión de trabajo que } \\
\text { aborde problemáticas reales } \\
\text { fuertemente vinculadas al } \\
\text { contexto profesional. }\end{array}$ \\
\hline
\end{tabular}

Como hemos visto, la práctica de los principios de la evaluación auténtica, implican una actuación estratégica y dosificada por parte del docente, en donde cada actividad de evaluación, debe plantearse en función del nivel de avance del estudiantado en el plan de estudios, es decir, la fase de formación profesional en la que un colectivo de estudiantes se encuentre cursando.

\section{A modo de conclusión}

A nivel general, una evaluación auténtica más allá de priorizar el uso de la memoria y mediciones cuantitativas, permite valorar de manera profunda, global y argumentada las decisiones y procedimientos empleados por un estudiante al resolver un problema muy similar a los que tendrá que resolver en su vida profesional.

En este proceso, han de movilizarse de manera coordinada un conjunto de saberes cognitivos, (lo que debo conocer) procedimentales, (lo que debo saber hacer) actitudinales, (lo que debo valorar) y estratégicos, (toma de decisiones), que garanticen el desarrollo de las competencias necearías en el aprendizaje de una disciplina.

De acuerdo con lo anterior, se puede inferir que los principios establecidos por la evaluación auténtica, resultan ser ciertamente 
compatibles con la enseñanza por competencias debido al carácter contextual y situado de ambos posicionamientos pedagógicos, en donde se privilegia una enseñanza, aprendizaje y evaluación del conocimiento profesional, tomando en consideración múltiples variables, vinculadas íntimamente a los diversos contextos y escenarios reales en los que dichos conocimientos han de ser aplicados.

Lo anterior nos lleva a hacer una revisión acerca de cuáles son las concepciones predominantes en los docentes universitarios con respecto a la evaluación de los aprendizajes y si valdría la pena aproximar dichas concepciones hacia posturas cercanas a la autenticidad, al aprendizaje situado y en general hacia principios socio constructivistas, lo cual permita al docente universitario conocer con mayor precisión como emprender un proceso de evaluación de los aprendizajes, que no se separe de su proceso de enseñanza ni de las demandas del contexto profesional.

\section{Referencias bibliográficas}

Coll, C. (2007). Las competencias en la educación escolar: Algo más que una moda y mucho menos que un remedio. Aula de innovación educativa, 161.34-39.

Coll, C. (2009). ¿Son los enfoques del aprendizaje basados en competencias, un instrumento útil para hacer frente a los desafios asociados a la pérdida relativa del sentido de la educación y del aprendizaje escolar? Recuperado de http://www.youtube.com/ watch? $\mathrm{v}=12 \mathrm{xaCoo}$ 09I,

Fernández, A. (2009). La evaluación orientada al aprendizaje en un modelo de formación por competencias en la educación universitaria. Revista de Docencia Universitaria, 8 (1), 11-34.

Gibbs, G. (1992). Assessing More Students. Oxford: Oxford Centre for Staff Development.

Gielen, S., Dochy, F. y Dierick, S. (2003). The influence of assessment on learning. En M. Monereo, C. (2009). La autenticidad de la evaluación en CASTELLÓ M. (Coord). La evaluación auténtica en enseñanza secundaria y universitaria, Barcelona, Edebé, Innova universitas.

Monereo, C. (2007). La evaluación auténtica de competencias: Posibles estrategias. Recuperadodehttp://www.sinte.es/carlesmonereo/?page_id=101 
Prodomou, L. (1995). The backwash effect: from testing to teaching, ELT Journal, 49(1), 13-25.

Savery J.R. y Duffy, T.M. (1995). Problem-based learning: An instructional model and its constructivist framework. In B. Wilson (Ed.), Constructivist learning environments: Case studies in instructional design (pp. 135-148). Englewood Cliffs, NJ: Educational Technology Publications.

Sweller, J., Van Merrienboer, J. J. G. y PAAS, F. G. W. C. (1998). Cognitive architecture and instructional design. Educational Psychology Review, 10, 251-296.

Wiggins, G. (1989). A True Test: Toward More Authentic and Equitable Assessment. The Phi Delta Kappan, 70 (9), 703-713.

Woolflok, A. (1999). Psicología Educativa. México: Prentice Hall.

Zabala, A. y Arnau, L. (2007). 11 ideas clave: Cómo aprender y enseñar competencias. Barcelona, Graó. 